\title{
RHINOLOGY
}

\section{Effectiveness of superior eyelid endoscopic-assisted approach in the management of selected orbital abscess: considerations on 4 cases}

\author{
Ruolo dell'approccio trans-palpebrale superiore endoscopico-assistito nella gestione \\ delle complicanze infettive orbitarie multicompartimentali: considerazioni su 4 casi clinici \\ lacopo Dallan, Lodovica Cristofani-Mencacci, Christina Cambi, Mariella Scarano, Augusto Pietro Casani, Veronica Seccia \\ Otorinolaringoiatria Audiologia e Foniatria, Azienda Ospedaliero-Universitaria Pisana, Pisa, Italy
}

\section{SUMMARY}

Orbital abscess is one of the most serious complications of acute rhinosinusitis and requires surgical management to avoid further complications such as visual loss and intracranial extension of the infectious process. Endoscopic sinus surgery is the most frequently used procedure in such cases, even if its use may be insufficient in some cases. The aim of the present study is to present our experience on the management of selected orbital abscesses through a superior eyelid endoscopic approach in combination with endonasal surgery. Personal considerations on four cases and lessons learned are described.

KEY WORDS: abscess, sinusitis, rhinitis, orbital cellulitis, endoscopy

\section{RIASSUNTO}

Gli ascessi dell'orbita rappresentano una delle più temibili complicanze in corso di rinosinusite acuta. La loro gestione è di tipo chirurgico nella maggior parte dei casi, con l'obiettivo di drenare la raccolta ed evitare l'evoluzione verso quadri ancora più seri che possano comportare la perdita della vista o l'estensione intracranica del processo infettivo. L'approccio chirurgico più frequentemente utilizzato è rappresentato dalla chirurgia endoscopica dei seni paranasali, pur risultando in alcuni casi insufficiente. Lo scopo del presente studio è quello di presentare la nostra esperienza nell'utilizzo dell'approccio trans-palpebrale superiore in combinazione con la via endoscopica endonasale classica nella gestione di casi selezionati di ascessi orbitari.

PAROLE CHIAVE: ascesso, sinusite, rinite, cellulite orbitaria, endoscopia

\section{Introduction}

Infectious orbital complications may occur secondary to acute rhinosinusitis, when pathogens spread from an infected sinus into the orbit. It may happen directly, given the close proximity of the orbit to the paranasal sinuses, or indirectly via the bloodstream. Chandler's classification for orbital complications of sinusitis distinguishes five stages on the basis of involvement of the orbital content ${ }^{1}$. Conservative management is possible in earlier Chandler's stages, while surgical drainage is indicated in later stages or in cases of failure to improve under medical treatment. The Chandler's IV stage is represented by an orbital abscess, in which purulent secretions are described within the orbital content, deep to the periorbita. Patients experience proptosis, chemosis, orbital pain and progressive ophthalmoplegia and, if not promptly treated, visual impairment and visual loss ${ }^{1}$. In this group of patients, surgical management is highly recommended in order to drain the abscess, release pressure in
Received:February 11, 2020

Accepted: May 20, 2020

\section{Correspondence}

Lodovica Cristofani Mencacci

Otorinolaringoiatria Audiologia e Foniatria, Azienda Ospedaliero-Universitaria Pisana, via Paradisa 2, 56124 Pisa, Italy

Tel. +39050997501

E-mail: lodovicacristofani@yahoo.it

Funding

None.

Conflict of interest

The Authors declare no conflict of interest.

How to cite this article: Dallan I, CristofaniMencacci L, Cambi C, et al. Effectiveness of superior eyelid endoscopic-assisted approach in the management of selected orbital abscess: considerations on 4 cases. Acta Otorhinolaryngol Ital 2020;40:421-425. https://doi. org/10.14639/0392-100X-N0679

(c) Società Italiana di Otorinolaringoiatria e Chirurgia Cervico-Facciale

\section{ल)(1)}

This is an open access article distributed in accordance with the CC-BY-NC-ND (Creative Commons Attribution-NonCommercial-NoDerivatives 4.0 International) license. The article can be used by giving appropriate credit and mentioning the license, but only for non-commercial purposes and only in the original version. For further information: https:// creativecommons.org/licenses/by-nc-nd/4.0/deed.en 
the orbit, obtain material for culture and reduce the risk of intracranial extension, including cavernous sinus thrombosis, meningitis, cerebritis and cerebral abscess (Chandler's stage $\mathrm{V})^{2}$. Endoscopic sinus surgery is the most frequently used procedure even if its use may be insufficient when dealing with areas that are complex to treat. In other words, the effectiveness of endonasal procedures depends on the location of the disease within the orbit, eventually requiring the association with or the necessity to switch to other approaches.

The aim of the present study is to present our experience on management of selected orbital abscesses through a superior eyelid endoscopic approach in combination with endonasal surgery. Personal considerations and lessons learned are described.

\section{Materials and methods}

In this retrospective cohort study, we report our experience with four adult patients treated with a superior eyelid transorbital endoscopic approach in combination with endonasal surgery for rhinogenic orbital abscesses at the University Hospital of Pisa between July 2016 and March 2019. Patients treated with an exclusive endonasal approach were not considered. Data collected included demographic, known history of chronic rhinosinusitis, previous nasal or orbital surgical procedures, ongoing medical treatments. All patients underwent a pre-operative eye examination and CT scan to evaluate orbital involvement (Fig. 1). All patients provided informed written consent for the procedure. Patients were selected for a superior eyelid endoscopic approach in combination with endonasal endoscopic surgery based on the location of the abscess within the orbit. In other words, the main criterion to indicate the need for a superior-eyelid approach was the location of the abscess in the supero-lateral orbital quadrant. Surgical procedures performed, hospitalisation days and complications were analysed. No statistical evaluations were performed due to the low number of patients.

\section{Notes on surgical technique}

The transnasal approach consisted in subtotal resection of the middle turbinate; complete spheno-etmoidectomy; exposure and removal of the lamina papyracea; periorbital exposure and opening and eventually extraconal fat management and drainage of the abscess. The transorbital approach was performed via superior eyelid incision and included identification of the orbicolaris oculi muscle; harvesting of the suborbicolaris flap and dissection until the orbital rim; dissection of the periorbita from the orbital bones and creation of enough room for instrumentation; incision of the

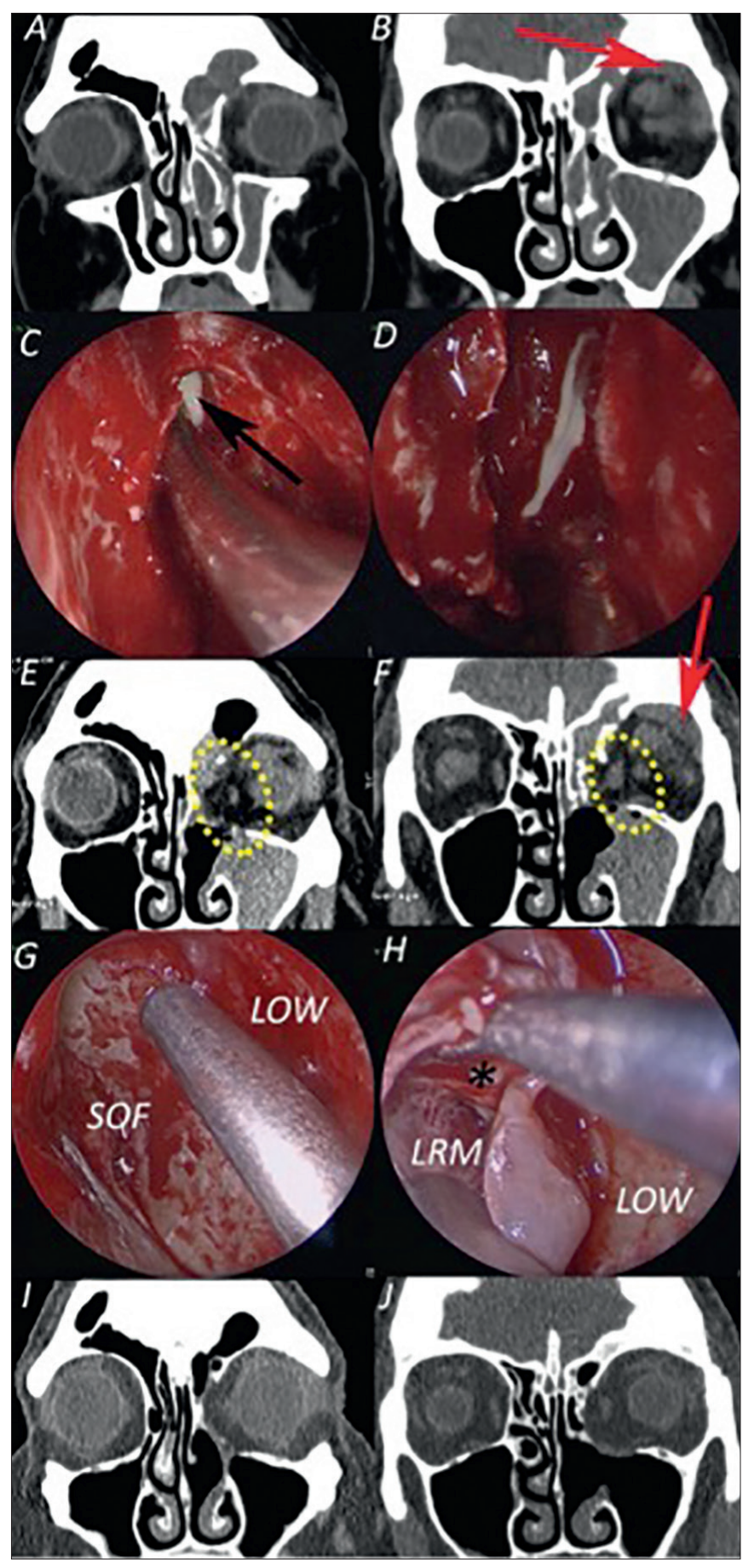

Figure 1. Radiological and endoscopic images describing the time evolution of the orbital abscess in patient number 1. Radiological evidence of a left acute rhinosinusitis (A, B). Red arrow show orbital roof dehiscence with involvement of the supero-lateral orbital compartment. Endoscopic evidence of pus during the endonasal surgical approach (black arrow in C, D). Post-operative radiological images showing adequate surgical outcomes of a complete ethmoidectomy with orbital decompression (E, F). Red arrow shows the persistence of the supero-lateral orbital abscess. Endoscopic images of the transorbital surgical approach (G, H). LOW: left orbital wall; SOF: superior orbital fissure; LRM: left rectus muscle; " shows the pus. Post-op radiological images showing adequate surgical outcome and complete resolution of the orbital abscess $(\mathbf{I}, \mathbf{J})$. 
periorbita and dissection within the orbital fat till abscess is reached. Drainage of the abscess was performed according to individual requirements. The drain was removed the day after surgery. Neuronavigation was used to confirm the exact position of the abscess and to help the surgeon identify the abscess.

\section{Results}

Four adult patients affected by sinogenic orbital abscess were treated with a combined transnasal-transorbital endoscopic surgical procedure. Our cohort was formed by 3 males and 1 female, with a mean age of $39.75 \pm 25$ years. All patients presented acute rhinosinusitis with acute onset of orbital symptoms. Two patients had a known history of chronic rhinosinusitis, managed with topical steroids and in one patient with previous surgery. All patients were on antibiotic therapy when they came to our attention. Clinical details of the patients are reported in Table I. All patients presented a Stage IV Chandler's classification for acute rhinosinusits complications, as revealed by pre-operative radiological evaluation. At pre-operative eye examination, all patients presented chemosis, proptosis and orbital pain; two patients presented visual impairment: deep reduction of visual acuity in patient \# 1; blindness in patient \# 2 (Tab. II).

Surgery was represented by a combined transnasal-transorbital approach in three of four patients; one patient was treated transnasally and transorbitally at different times (patient \# 1). Patient \# 4 developed an asymptomatic epidural abscess in the post-operative period that was successfully treated with medical therapy in the infectious diseases ward. Long-term sequalae occurred in patient \# 2 in terms of lack of improvement of blindness. All perioperative details are reported in Table III.

\section{Discussion}

Orbital abscess is one of the most frightening complications of acute rhinosinusitis and should be considered a very serious condition with possible permanent sequelae. In case of orbital abscesses, surgery should become rapidly advisable, although conservative management could be attempted in selected adult patients with limited subperiosteal

Table I. Clinical details of our cohort of patients.

\begin{tabular}{lccccc} 
Patient number & Sex, Age (years) & Known CRS & Previous surgery & Days from onset of ARS & Ongoing antibiotic treatment \\
1 & F, 75 & Y & N & 9 & Amox/Clav Ac. \\
2 & M, 40 & Y & Y & 6 & Cefixime-Amox/Clav. Ac. \\
3 & M, 27 & N & N & 1 & Amox/Clav. Ac. \\
4 & M, 18 & N & N & 9 & Levoflox-Ceftriaxone \\
\hline
\end{tabular}

M: male; F: female; Known CRS: known history of chronic rhinosinusitis; Days from onset of ARS: days between onset of acute rhinosinusitis and onset of orbital symptoms; Amox/ Clav. Ac.: Amoxicillin/Clavulanic Acid; Levoflox: Levofloxacin

Table II. Ocular symptoms.

\begin{tabular}{lccccc} 
Patient number & Chemosis & Proptosis & Visual impairment & Ocular motility impairment & Orbital pain \\
1 & $Y$ & $Y$ & $Y, 1 / 10$ & $Y$ & $Y$ \\
2 & $Y$ & $Y$ & $Y, 0 / 10$ & $Y$ & $Y$ \\
3 & $Y$ & $Y$ & $N, 8 / 10$ & $\mathrm{~N}$ & $\mathrm{Y}$ \\
4 & $Y$ & $Y$ & $\mathrm{~N}, 10 / 10$ & $\mathrm{Y}$ \\
\hline
\end{tabular}

Table III. Perioperative details, complications and sequalae in our cohort.

\begin{tabular}{lcccccc}
$\begin{array}{l}\text { Patient } \\
\text { number }\end{array}$ & Surgery performed & Days of hospitalisation & Post-op CT scan & Second surgery & Short-term sequalae & Long-term sequalae \\
\hline 1 & Transnasal & 10 & OA & Y, Transorb & $\mathrm{N}$ & $\mathrm{N}$ \\
2 & Combined & 6 & $\mathrm{Neg}$ & $\mathrm{N}$ & Blindness \\
3 & Combined & 10 & $\mathrm{Neg}$ & $\mathrm{N}$ & $\mathrm{N}$ & $\mathrm{N}$ \\
4 & Combined & Other dept. & EA & $\mathrm{N}$ & $\mathrm{N}$ \\
\hline
\end{tabular}

Transnasal: Transnasal endoscopic surgery; Combined: Transnasal-transorbital endoscopic surgery; Transorb: Transorbital endoscopic surgery; Other dept: moved to other department; OA: orbital abscess; Neg: negative for persistence of abscess or other complication; EA: Epidural abscess 
abscesses ${ }^{3}$. Time is typically a critical factor, and, in such "conservative" cases, patients need to be closely monitored in order to avoid possible evolution and thus serious complications ${ }^{4}$. Technically speaking, most of subperiosteal abscesses can be managed transnasally. This is not always true in all cases of orbital abscesses. Thus, if endoscopic endonasal surgery is adequate in most cases, with the aim of opening the sinuses involved, removing pus, allowing direct cultures and providing a drainage pathway, this approach may be insufficient in cases of abscesses involving the supero-lateral orbital compartments. These orbital areas are normally involved due to spread of infection, although septic emboli may occur ${ }^{5}$. Hence, in suspected orbital abscesses orbital imaging becomes mandatory to confirm the suspicion and to choose the most appropriate approach. If MRI with contrast is the best option to evaluate orbital lesions, it is quite clear that examination is rarely available in such an urgent situation. Therefore, the vast majority of these cases have to be managed using CT, if possible using a contrast agent. We strongly advise, in such a scenario, to prefer the rapidity of action in respect to searching for the best imaging examination. Since most orbital abscesses are related to acute rhinosinusitis, we consider, as stated before, that the endonasal approach should be the first procedure to be performed in order to drain the purulence and restore ventilation to the sinuses ${ }^{3}$. On the other hand, there are clear limitations for an exclusive endonasal approach in managing selected sinogenic orbital complications, especially in case of supero-lateral or lateral abscesses. In these cases, transnasal approaches offer an unfavourable path-to-target trajectory, while other routes, like the superior eyelid approach, could be more efficient. Practically speaking, in these complex cases, it could be necessary to combine endonasal and transorbital approaches, and this is what we did in our patients. We normally move first with a transnasal approach and then a transorbital approach if a supero-lateral abscess is demonstrated by imaging. In our first case, imaging seemed to show a sub-periosteal abscess extending from the medial orbital compartment to the superior ones. Thus, we performed, as a first surgical procedure, an exclusive transnasal approach, although intraoperatively, sub-periosteally, there was only a limited amount of infected material. At post-operative CT, an abscess was still demonstrated in the supero-lateral orbital compartment, thus requiring a further surgical procedure via superior eyelid (Fig. 1). Since that time, we shifted to a combined procedure in case of supero-lateral orbital involvement. The effectiveness of the superior eyelid approach in the setting of intraorbital abscesses has been previously reported by other authors ${ }^{5}$. In fact, the superior eyelid approach is not thought to replace transnasal or other approaches, but rather to serve as a complementary tool to help the surgeon when facing challenging areas. Despite the high-risk anatomic location of the abscesses in our series, we have documented the feasibility and excellent safety profile of the superior eyelid approach. All abscesses were successfully reached and drained as intended (Fig. 2). In our series there were no new visual deficits, worsened diplopia, or ptosis.

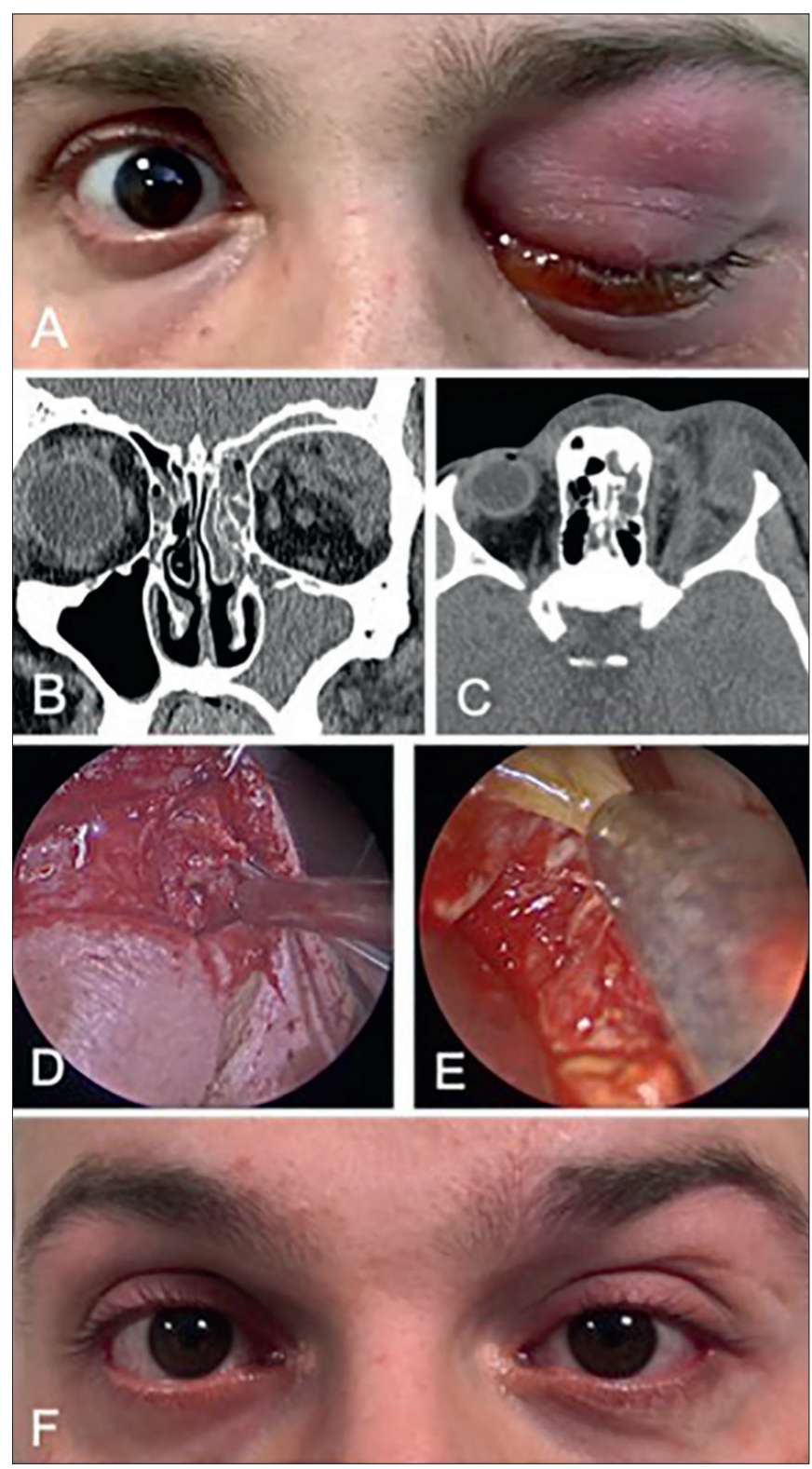

Figure 2. Clinical, radiological and surgical images of a left orbital abscess. Clinical presentation with significant proptosis, chemosis and inability to open the eye (A). Coronal and axial CT scan images showing the left orbital involvement in the context of a left acute rhinosinusitis $(\mathbf{B}, \mathbf{C})$. Endoscopic evidence and drainage of pus during the transorbital approach (D, E). Post-operative image showing complete resolution of the proptosis and chemosis. There is no evidence of enophthalmos $(\mathbf{F})$. 
Additionally, we did not experience unfavourable wound healing, eyelid distortion, or poor cosmetic outcomes associated with the superior eyelid incision. Patient \# 2 in our cohort unfortunately experienced permanent vision loss, which was likely related to delayed surgery (about 24 hours after onset). This patient was blind before surgery and vision did not recover. Although anecdotical, this stresses the importance of rapid management of such abscesses, considering that delayed surgery may be associated with a reduced chance of visual recovery and an increased risk of intracranial complications. In this context, secondary vascular damage (mostly venous related) of minor but critical orbital vessels could be responsible of the dramatic worsening of the vision. Lastly, in our opinion, the transorbital route should be considered a minimally invasive, safe and effective approach to be further developed in combination with transnasal surgery. Its role in the management of orbital abscess should be considered based on the site and features of the abscess by CT. On these grounds, the transorbital endoscopic approach may overcome the limits of the transnasal route, providing improved visualisation and access to challenging anatomical areas. From a clinical point of view, we strongly advise careful and rapid evaluation of this patients, and if a supero-laterally located abscess is suspected a combined transnasal-transorbital procedure should be considered.

\section{Conclusions}

We present the transorbital endoscopic approach as an effective route to use in combination with the transnasal approach in cases of orbital abscess that involve multiple, not-necessarily-communicating compartments. More practically, the involvement of the supero-lateral compartment is a strong recommendation for the superior eyelid approach. In this scenario, only accurate pre-operative radiological evaluation will allow choice of the most appropriate surgical route.

\section{References}

1 Chandler JR, Langenbrunner DJ, Stevens ER. The pathogenesis of orbital complications in acute sinusitis. Laryngoscope 1970;80:141428. https://doi.org/10.1288/00005537-197009000-00007

2 Howe L, Jones NS. Guidelines for the management of periorbital cellulitis/abscess. Clin Otolaryngol Allied Sci 2004;29:725-8. https:// doi.org/10.1111/j.1365-2273.2004.00889.x

3 Gavriel H, Jabrin B, Eviatar E. Management of superior subperiosteal orbital abscess. Eur Arch Otorhinolaryngol 2016;273:145-50. https:// doi.org/10.1007/s00405-015-3557-1

4 Harris GJ. Subperiosteal abscess of the orbit: older children and adults require aggressive treatment. Ophthalmic Plast Reconstr Surg 2001;17:395-7. https://doi.org/10.1097/00002341-200111000-00002

5 Lim JH, Sardesai MG, Ferreira M Jr, et al. Transorbital neuroendoscopic management of sinogenic complications involving the frontal sinus, orbit, and anterior cranial fossa. J Neurol Surg B Skull Base 2012;73:394-400. https://doi.org/10.1055/s-0032-1329617 